\title{
In-flight calibration of the Cluster PEACE sensors
}

\author{
N. Doss ${ }^{1}$, A. N. Fazakerley ${ }^{1}$, B. Mihaljěici ${ }^{1}$, A. D. Lahiff ${ }^{1, *}$, R. J. Wilson ${ }^{1, * *}$, D. Kataria ${ }^{1}$, I. Rozum ${ }^{1, * * *}$, G. Watson ${ }^{1}$, \\ and Y. Bogdanova ${ }^{1, *}$ \\ ${ }^{1}$ Mullard Space Science Laboratory, University College London, Dorking, UK \\ *now at: Rutherford Appleton Laboratory, Oxford, UK \\ *** now at: University of Colorado Boulder, Colorado, USA \\ **** now at: European Centre for Medium-Range Weather Forecasts, Reading, UK
}

Correspondence to: N. Doss (n.doss@ucl.ac.uk)

Received: 29 July 2013 - Published in Geosci. Instrum. Method. Data Syst. Discuss.: 10 October 2013

Revised: 10 February 2014 - Accepted: 12 March 2014 - Published: 5 May 2014

\begin{abstract}
The Plasma Electron and Current Experiment (PEACE) instruments operate on all four of the Cluster spacecraft and measure the 3-D velocity distribution of electrons in the energy range from $0.59 \mathrm{eV}$ to $26.4 \mathrm{keV}$ during each spacecraft spin. Pitch angle distributions and moments of the velocity distribution are also produced. As the mission has progressed, the efficiency of the detectors has declined. Several factors may play a role in this decline such as exposure to radiation, high electron fluxes and spacecraft thruster firings. To account for these variations, continuous in-flight calibration work is essential. The purpose of this paper is to describe the PEACE calibration parameters, focussing in particular on those that vary over time, and to describe the methods which are used to determine their evolution.
\end{abstract}

\section{Introduction}

A detailed description of the Plasma Electron and Current Experiment (PEACE) instrument is not provided here but can be found in Johnstone et al. (1997) and Fazakerley et al. (2010a).

Each of the Cluster spacecraft carries an identical PEACE instrument which consists of two sensors and a data processing unit. Both sensors are capable of covering the full energy range of the instrument, but each sensor usually covers about $70 \%$ of the instrument energy range in any given spin. The LEEA (Low Energy Electron Analyser) sensor has a smaller geometric factor appropriate for the higher fluxes that are normally found at the lower energies such as in the solar wind and magnetosheath. The HEEA (High Energy Electron Anal- yser) sensor has a larger geometric factor better suited for the weaker fluxes seen in the magnetosphere. Used together the sensors can cover the full energy range every spin.

Each of the sensors is a "top hat" electrostatic analyser (Carlson et al., 1982), whose operational principle is illustrated in Fig. 1. A voltage is applied across the hemispheres of the analyser which diverts electrons of a specific energy and acceptance angle (shown in blue) through the analyser to the semi-annular micro-channel plate (MCP) detector. Sunlight passes through the aperture and out again; measures are taken to minimise the amount of light reflected within the analyser and reaching the MCP. Electrons which do not have the selected energy (shown in red) strike the analyser hemispheres and are not counted. When an electron reaches the MCP, the signal is amplified and the resulting charge cloud is detected in one of the 12 segments of the anode beneath, providing information about the direction in which the electrons were travelling. The number of electrons that result for each incident electron is defined as the gain of the MCP. A voltage is applied across the $\mathrm{MCP}$ in order to produce charge amplification.

The two PEACE sensors are mounted on opposite sides of the spacecraft with their field-of-view fans lying perpendicular to the spacecraft surface as illustrated in Fig. 2. The field of view of each PEACE sensor perpendicular to the spacecraft frame is $3.8^{\circ}$ (HEEA) and $2.9^{\circ}$ (LEEA). The azimuthal angle is measured in the spacecraft spin plane, while the polar angle is measured in the plane orthogonal to the spin plane. Each individual sensor has an $180^{\circ}$ field of view and covers a $4 \pi$ field of view in one spacecraft spin. The combined field of view of the two sensors covers the complete 


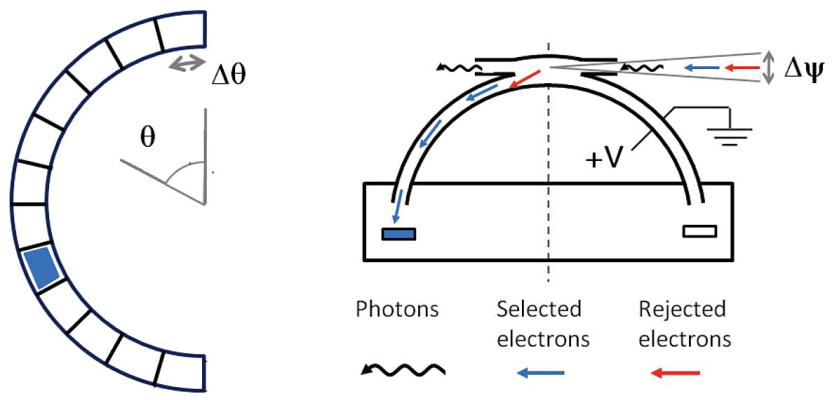

Fig. 1. Illustration of the principle of the PEACE electrostatic analyser.

$4 \pi$ solid angle range during half a spacecraft spin, in the energy range overlapped by the two sensors. The sensor numbering in Fig. 2 shows the direction from which the arriving electrons are counted on the 12 anodes (0 to 11$)$; e.g. zone 0 looks toward $-X_{b}$ and sees electrons travelling with velocities along the spin axis direction $+X_{b}$.

\section{The calibration parameters}

During a spin each PEACE sensor can sample the velocity distribution of the plasma electrons by making a series of individual measurements in a set of different look directions and energies. Such a measurement gives the velocity space density of the electrons, $f_{i j k}$, in the small region of velocity space defined by polar angle $i$, azimuthal angle $j$ and energy (speed) interval $k$. The velocity space density is related to measured quantities and calibration factors as follows:

$f_{i j k}=\frac{P_{i j k}}{t_{\mathrm{acc}} v_{k}^{4} G_{i} \varepsilon_{i k}}$,

where $P_{i j k}$ is the number of electrons counted after dead time correction (related to instrument electronics not the MCP); $t_{\mathrm{acc}}$ is the data accumulation time, a fixed fraction of the spin period; $v_{k}$ is the mean value of the measured electron speed during time $t_{\text {acc }} ; G_{i}$ is the geometric factor for the $i$ th polar angle sector, which in a perfectly concentric analyser reduces to a single value $G$ for all sectors (the geometric factor is different for HEEA and LEEA sensors due to different mechanical designs for the electrostatic analyser entrance aperture and collimator); and $\varepsilon_{i k}=\varepsilon_{0} \varepsilon\left(v_{k}^{2}\right)_{i}$ is the detector efficiency, which varies with time, position on the detector and electron energy. It is defined as the probability that a particle reaching the detector is actually registered. $\varepsilon_{0}$ is an energy- and position-independent efficiency term, and $\varepsilon\left(v_{k}^{2}\right)_{i}$ is the relative sensitivity of the detector as a function of position (i.e. anode segment) and electron energy.

\subsection{Ground calibration}

Ground calibration work established values for the non-timevarying parameters for each individual sensor before launch.

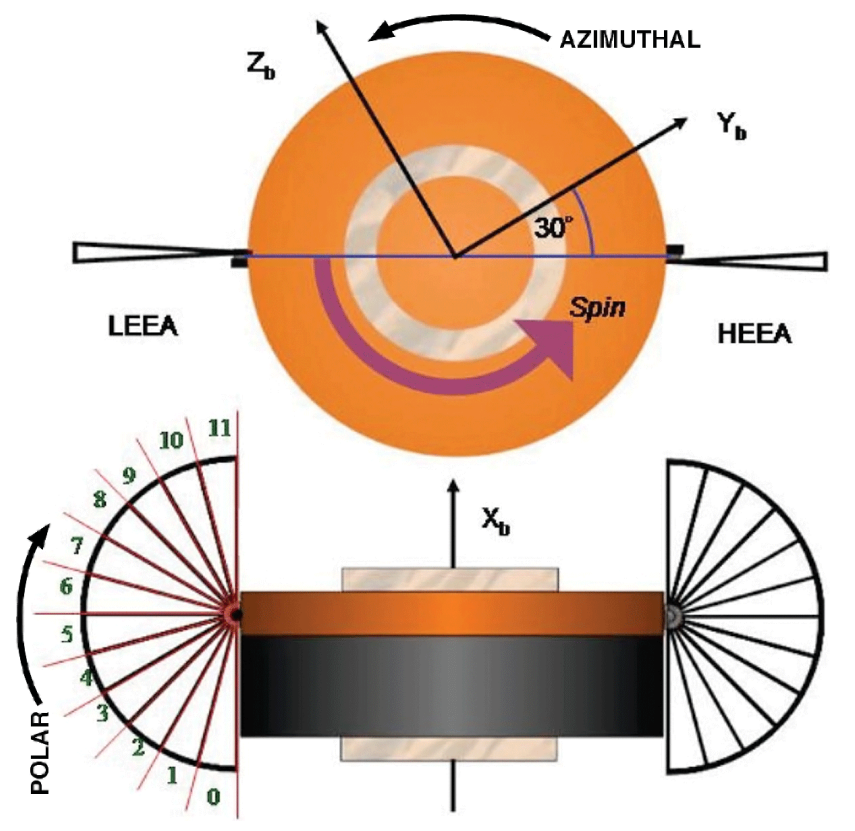

Fig. 2. The physical deployment of the PEACE LEEA and HEEA sensors on the spacecraft. The spacecraft body co-ordinate system is shown $\left(X_{b}, Y_{b}, Z_{b}\right)$. In orbit the spacecraft spin axes are maintained roughly anti-parallel to the GSE $z$ axis.

The electrostatic analysers of the four HEEA sensors were made as mutually identical as possible, similarly for the four LEEA sensors. All eight sensors use the same equipment to control the electron energy selection and to count detected electrons. The least controllable aspect of the design is the efficiency of the individual MCP detectors in each sensor. Values were obtained for the geometric factor $G_{i}$, the energies measured during energy sweeps $v_{k}$, and the relative sensitivity of the detector $\varepsilon\left(v_{k}^{2}\right)_{i}$, under conditions of optimum detector performance. Due to the difficulty in measuring the current in an electron beam with sufficient accuracy it is challenging to establish good values for $\varepsilon_{0}$ in a calibration facility. Therefore values for $\varepsilon_{0}$ were determined in flight through cross-calibration of PEACE and WHISPER (Waves of High Frequency and Sounder for Probing of Density by Relaxation) density measurements (Fazakerley et al., 2010b).

These parameters were measured in a test chamber to ensure the sensors were identical to within specified tolerances before being accepted for flight. The values obtained are used as the baseline from which any in-flight calibration corrections are made.

\subsection{Calibration correction factors}

It was expected that the performance of the detector would vary over time due to degradation of the MCP and thus require correction through in-flight calibration. There are two aspects of the instrument calibration which may vary during flight operations. The energy/angle-dependent detector 
efficiency, $\varepsilon_{i k}$, can be described by correction factors $(\alpha, \beta)$ that account for these variations over time as follows:

$\varepsilon_{i k}=\alpha(t) \varepsilon_{0} \beta_{i k} \varepsilon\left(v_{k}^{2}\right)_{i}$,

where $\alpha(t)$ is the time-dependent correction factor for the energy/angle-independent part of $\varepsilon_{i k}$. It describes the effect of the sub-optimal detector sensitivity as it declines over time. By definition this correction factor applies equally for all anodes.

$\beta_{i k}$ represents corrections for each anode to $\varepsilon\left(v_{k}^{2}\right)_{i}$, the relative sensitivity of the 12 individual anodes of each detector. Even small errors in the inter-anode calibration can result in large errors in the spin axis component of the bulk velocity which are determined by integration of the velocity space density collected during a spin. This correction factor varies with gain of the MCP.

The parameters $\alpha$ and $\beta$ were each equal to 1 in ground test conditions, when the MCP gain was well above $2 \times 10^{6}$ electrons.

It is possible to increase the detector efficiency by increasing the voltage applied across the MCP, and this has been performed in small steps periodically throughout the mission.

In this paper we describe two methods which have been used for determining how the $\alpha$ factor term changes throughout the mission. The first method, detailed in Sect. 3, uses data from weekly in-orbit tests of the PEACE sensor performance. However over time, some of the PEACE MCPs have degraded to the point that we cannot collect the input information needed to apply this method, and an alternative technique was developed. This second method uses comparisons of electron densities measured by PEACE LEEA and by other instruments to determine $\alpha$ for the LEEA sensor, and comparisons of PEACE HEEA and PEACE LEEA to determine $\alpha$ for the HEEA sensor. The latter method is currently in use and is described in Sect. 4 . We also present the results of these studies of the detector sensitivity evolution and comparisons of alpha determined by both methods.

The method for determination of the $\beta$ correction factors has been described elsewhere (see Fazakerley et al., 2010b) and not provided here.

\subsection{Relationship between calibration factors and moments}

It can be shown that the electron density measured by PEACE is inversely proportional to the calibration factors $G \alpha \varepsilon_{0}$. Thus these calibration factors need to be well characterised to achieve good densities from PEACE. It also has a more complex dependency on $\beta_{i k} \varepsilon\left(v_{k}^{2}\right)_{i}$. In our experience so far the required correction to $\alpha$ is usually much greater than $\beta_{i k}$. The effect of the $\beta_{i k}$ correction on the density has been checked after $\beta_{i k}$ is applied by repeating the $\alpha$ work. The subsequent correction to $\alpha$ was found to be very small.

The electron bulk velocity is independent of $G \alpha \varepsilon_{0}$ but has a strong dependency on $\beta_{i k} \varepsilon\left(v_{k}^{2}\right)_{i}$; therefore to achieve good plasma bulk flow velocity vectors requires accurate determination of the relative sensitivity of the 12 detector polar zones as a function of energy and gain. The independence of velocity from $G \alpha \varepsilon_{0}$ means that $\beta_{i k} \varepsilon\left(v_{k}^{2}\right)_{i}$ can be determined independently from $G \alpha \varepsilon_{0}$ using the method described in Fazakerley et al. (2010b). The validity of $\beta_{i k}$ corrections can be confirmed by comparing PEACE velocity measurements with CIS (Cluster Ion Spectroscopy experiment) (Rème et al., 2001) velocity measurements.

\section{Determination of $\alpha$ using in-orbit MCP tests}

\subsection{MCP gain-voltage characteristic before launch}

The gain for each individual MCP as a function of the voltage applied was characterised in ground calibration tests prior to launch. Using a radioactive tritium source which has a wellknown emission rate, the flux of beta particles (i.e. electrons which have a maximum energy of $18.6 \mathrm{keV}$ ) was measured while operating across a range of MCP voltage levels. At a given MCP voltage the number of electrons emerging from the MCP in response to an incident electron is not always the same. The spread of values of measured charge produced by the MCP for a given voltage is characterised by a pulse height distribution (PHD) as shown by the sketch in Fig. 3. As the voltage increases, larger signals are generated and the PHD peak moves to higher gains. From the ground test results, the peak of each pulse height distribution was taken to be the modal gain for that voltage. Using these points a characteristic gain-versus-voltage curve, as illustrated by the black curve in Fig. 4, was produced for each MCP. The PEACE instruments have a threshold level, $\sim 0.45 \times 10^{6}$ electrons, below which the counter electronics ignore the signal. The voltage at which the PHD modal gain is equal to the threshold level is referred to as the threshold voltage, shown by $V_{\text {ref }}$ in Fig. 3 .

\subsection{MCP gain evolution monitoring: dual-sensor technique}

We choose to operate the MCPs with a specific operational voltage level in order to achieve a desired gain. The MCP efficiency at a given voltage decreases with increased operating time. We compensate for the performance decline of the MCP over time by periodically raising the operational voltage level applied across the analyser to maintain the desired gain. In between these periodic voltage level raises we need to correct for the sub-optimal detector sensitivity, which requires us to know how the MCP is performing at a specific time. It is not possible to perform in-orbit tests in which we measure PHDs directly, so an alternative method was required. A unique technique was developed to estimate the PHD modal gain though not the full PHD shape. The technique makes use of the fact that we have two sensors on each spacecraft and is based on two assumptions: firstly, 


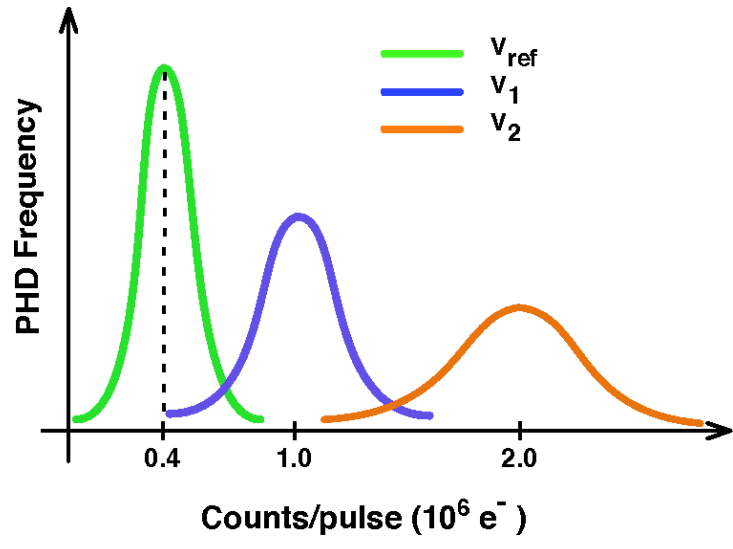

Fig. 3. Sketch of the pulse height distributions (PHDs) of measured values of charge from tritium tests for different voltages applied across the MCP during ground calibration tests. The peak of the PHD gives the modal gain for that voltage. As the voltage across the MCP is raised, e.g. from $V_{1}$ to $V_{2}$, the modal gain also increases. The increase in PHD spread with voltage is characteristic MCP behaviour. The ratio of the PHD FWHM to the PHD peak is typically observed to be roughly constant; thus as the voltage increases, so does the spread.

that the PHD for any given voltage is symmetric about the modal gain, and, secondly, that the gain-voltage curve does not change shape significantly as the MCP ages. The first assumption is based on measurements of the PHDs for the sensors during ground tests. The PHDs were typically neither perfectly symmetric nor far from being symmetric. They also varied slightly from sensor to sensor, and as a function of gain. Nonetheless, in a normal operating regime we consider that the assumption of symmetry is a good first approximation. The second assumption is based in part on literature that shows examples of similar MCPs that have been tested before and after "scrubbing", e.g. Eberhardt (1979). Our assumptions also seems to us to be well justified by the agreement between alpha factors determined using our technique and those inferred via density comparisons with WHISPER. At any given time the peak of the PHD moves to higher gains with increasing voltage level (Fig. 5a). As the modal gain rises, the fraction of the PHD which lies above the electronic threshold of the instrument, and so the fraction registered by the counter electronics, increases (Fig. 5b). The fraction of the PHD above the electronic threshold gives the efficiency of the MCP. In the case of perfectly symmetric PHDs, the position of the point of inflection on the "cumulative distribution function above threshold" curve is where the MCP is counting $50 \%$ of the electrons entering its pores, and so the gain can be determined and is equal to the electronic threshold.

An in-orbit MCP test procedure was created where we can apply the principle illustrated in Fig. 5. During a test both sensors, HEEA and LEEA, are set to observe the same energy range and thus the same plasma electrons. The MCP on

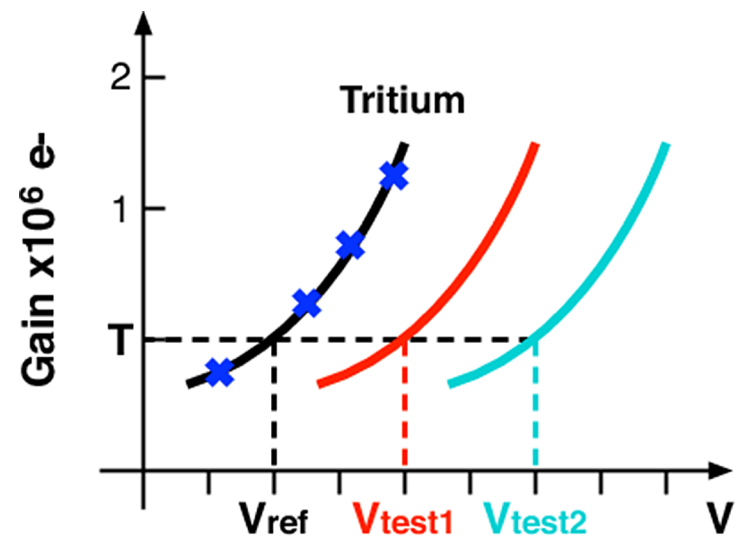

Fig. 4. Sketch of the characteristic gain-voltage curve. The black curve is the trend produced from the tritium tests prior to launch, where the blue crosses are the modal gains for particular voltages and $V_{\text {ref }}$ is the threshold voltage that has a corresponding gain equal to the electronic threshold of the instrument, $T$. The red and cyan curves show the gain-voltage curves shifted to higher threshold

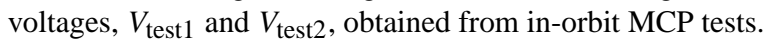
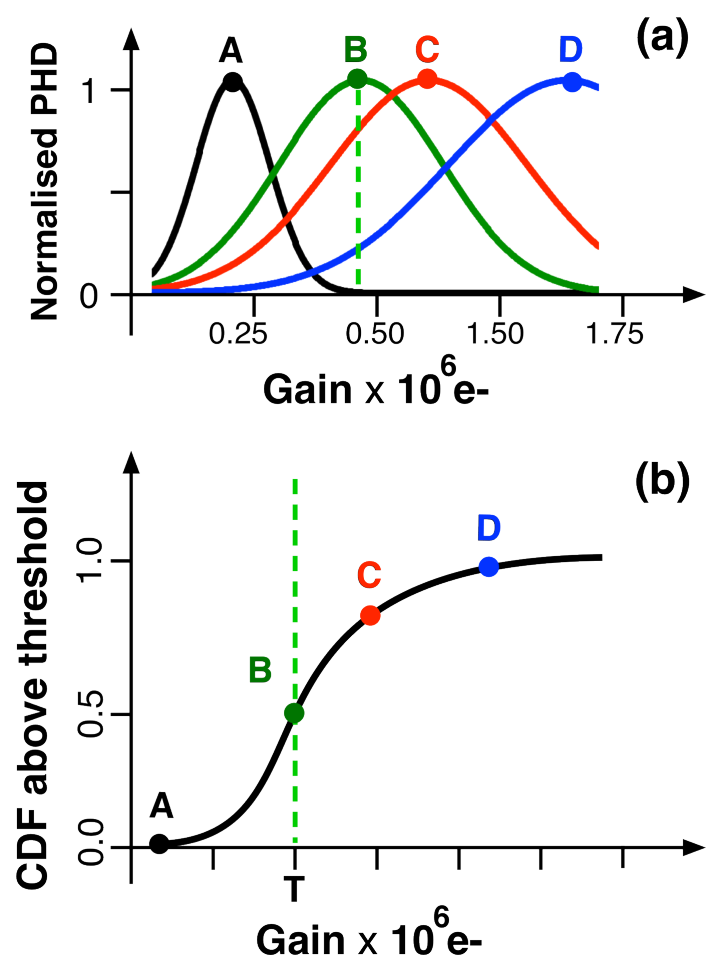

Fig. 5. Sketch of the normalised PHD and cumulative distribution function above threshold. Panel (a) shows how the normalised PHD changes for increasing modal gain, asymptotically approaching 1. Panel (b) shows the fraction of the PHD that lies above the electronic threshold of the instrument, shown by the vertical green dashed line, assuming a PHD symmetric about the peak. 

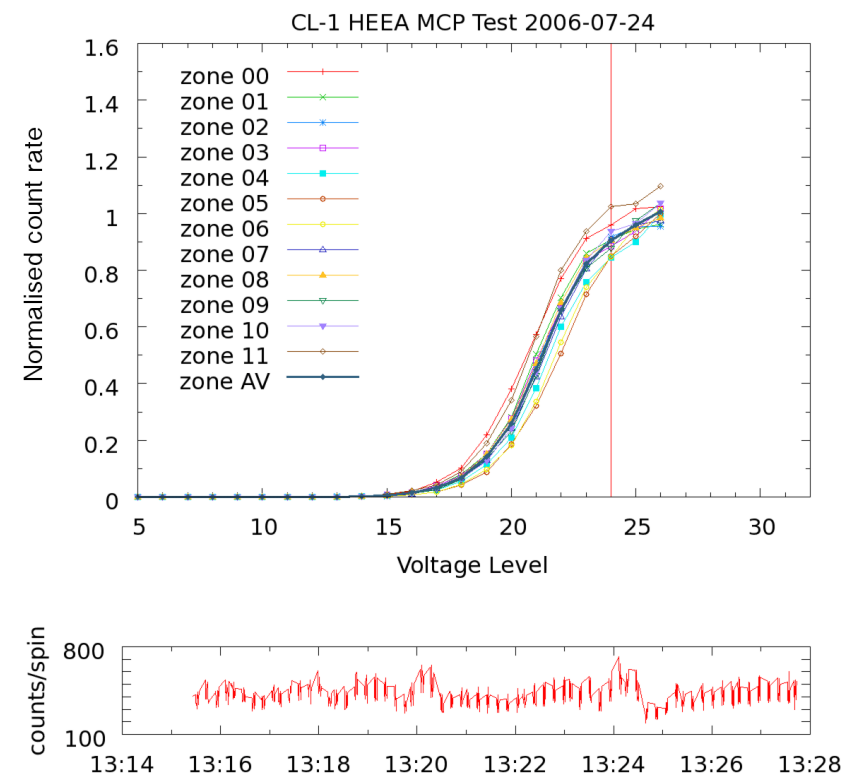

Fig. 6. Typical MCP test result for the Cluster-1 HEEA sensor. The upper panel shows the count rate from the HEEA (test) sensor collected over a range of MCP voltages. The count rates have been normalised using the count rates from the LEEA (monitoring) sensor shown in the lower panel. Curves are provided for each anode together with an average (thick dark blue line).

one sensor is swept through a range of voltage levels, sitting at each level for around $40 \mathrm{~s}$ (10 spacecraft spins), and the counts per spin at each level are measured for each anode. Simultaneously the other sensor is kept at a suitable voltage level in order to monitor variations of the plasma environment. The key advantage of this two-sensor technique is that the response of the measured count rate to increasing MCP voltage in the test sensor can be separated from the measured count rate changes due to flux variations in the ambient plasma, using data from the monitoring sensor. The test is then repeated for the other sensor by exchanging their roles. A typical weekly MCP test result is shown in Fig. 6 (for C1 HEEA). The upper panel shows the count rate from the HEEA (test) sensor collected over a range of MCP voltages. The count rates have been normalised using the count rates from the LEEA (monitoring) sensor shown in the lower panel. In this case the ambient plasma fluxes seen by the LEEA sensor are varying (bottom panel), showing the importance for normalisation. Curves are provided for each anode together with an average (thick dark blue line).

We assume a symmetrical PHD distribution, in which case the point of inflection on the averaged test curve gives the voltage $V_{\text {ref }}$ at which the gain is equal to the electronic threshold, the threshold voltage, at the time of the test. We now need to determine the gain at the normal operating MCP voltage, which is not usually the same as the threshold voltage. The vertical red line in Fig. 6 shows the normal operating voltage level of the HEEA sensor at the time of that test.

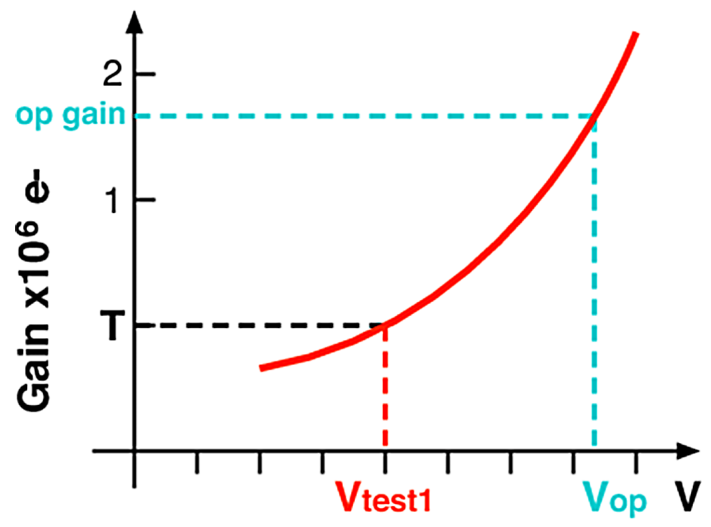

Fig. 7. Sketch of obtaining the MCP gain for the normal operational voltage level, $V_{\mathrm{op}}$, after shifting the characteristic gainvoltage curve to apply at the time of the weekly MCP test by requiring that the gain $=T$ when the MCP voltage equals the threshold value determined from the test.

To determine what the gain is for the operational voltage level, we apply our second assumption that the characteristic voltage-versus-gain curves obtained from the ground calibration tests do not change in shape over time but can simply be shifted to higher voltages, as shown by the red and blue curves in Fig. 4. We know that higher voltages are required to produce the same gain as the MCP ages, so our assumption is the simplest way to address this. We shift the curve so that the voltage $V_{\text {ref }}$ lines up with the threshold voltage obtained from the test, and we can then infer the gain at the time of the test for any other voltage level as shown in Fig. 7.

Once we know the gain of the MCP corresponding to the normal operational voltage level, we can use the cumulative distribution function (CDF) curve to determine what proportion of the PHD distribution lies above the electronic threshold for that value of gain (Figs. 8 and 5). This fraction is represented in our calibrations as the $\alpha$ factor, which is a measure of the number of electrons entering the MCP which are above threshold and thus are being counted.

In early calibration releases we used the mathematically derived Gaussian model of the cumulative distribution function shown by the red curve in Fig. 9. This worked well for high gains, which we verified by comparing PEACE densities with WHISPER densities; however it did not work so well at low gains $(<0.6)$. A new alpha factor model curve was created by using results from in-orbit MCP tests. An empirical curve was fitted to data of the normalised counts ratios from a large number of MCP tests, both HEEA/LEEA and LEEA/HEEA as shown in Fig. 9. This empirical alpha factor model deviates from the Gaussian model at lower gains, and it was found to give better results and is now used for calibrations instead of the Gaussian model. It was also used to recalibrate the earlier data sets. A single empirical curve was made for all spacecraft sensors; however future work is planned to check if different sensors can be better 


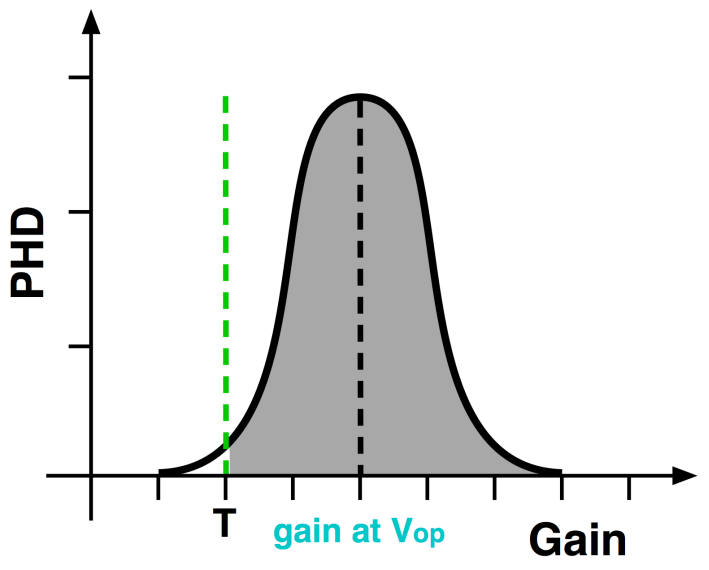

Fig. 8. Example sketch of the fraction of the PHD lying above the electronic threshold of the instrument, $T$, shown by the shaded area. The position of the peak of the PHD is at the gain corresponding to the normal operational voltage level, $V_{\mathrm{op}}$.

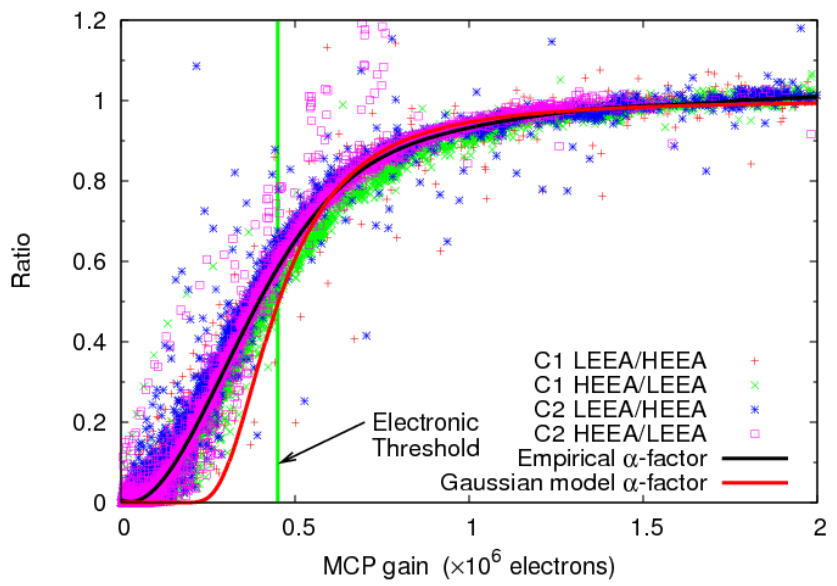

Fig. 9. Alpha-versus-gain curve. This plot shows the difference between the Gaussian model (red) and the empirical model (black) obtained from fitting to counts ratios from a large number of MCP tests, normalised to 1 at high gains.

characterised using individual curves. It may also be the case that the actual PHDs at low gains are not only not Gaussian but also not symmetrical, in which case our method may be less reliable as its assumptions are no longer completely applicable.

\subsection{Limitations of the dual-sensor in-orbit MCP test method}

In-orbit MCP tests are routinely carried out every week. An analysis of these tests gives a detailed time history of the detector sensitivity variations for each sensor. A time history of the threshold voltage determined from MCP test curves for the eight sensors is shown in Fig. 10.

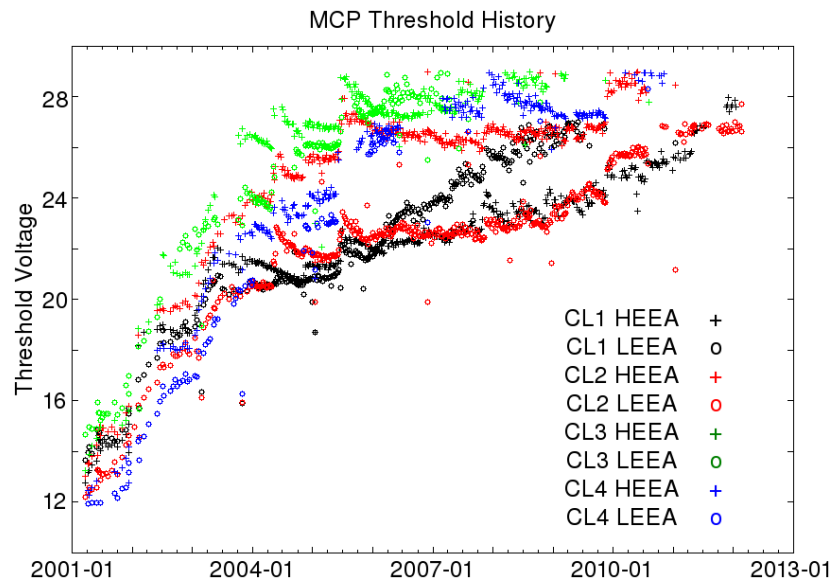

Fig. 10. Time history of the threshold voltage obtained from weekly MCP tests for all eight sensors up to 2012.

At the time of writing this method has ceased to be effective for the majority of the sensors. As noted above, we compensate for the efficiency decline of an MCP over time by periodically raising the operational voltage level applied across the MCP. Similarly, we have to use a set of voltage levels during the MCP tests that have higher voltage values than earlier in the mission, to produce the range of gain values that we wish to cover for the test, as illustrated in Fig. 5. The decline in efficiency has developed more rapidly across some sensors than others, and so the number of required voltage level increases has varied from sensor to sensor. On some sensors the highest available voltage level is now in use during normal operations, so it is no longer possible to raise them further. Also the threshold voltage is now close to the maximum level, so it is no longer possible to obtain a complete MCP test curve and identify the point of inflexion. This evolution is shown for two sensors in Fig. 11. These plots demonstrate the more rapid efficiency decline of the Cluster3 LEEA sensor in comparison to the Cluster-1 HEEA sensor. By 2006 C3 LEEA is already operating at one of the highest levels and the full curve is unobtainable. By 2012 this is also the case for C1 HEEA. In 2012 C3 LEEA is operating at its highest MCP voltage level, so compensating for efficiency decline in the future is no longer possible.

Although we no longer use this method for calibration purposes, we still routinely perform the weekly tests as we expect that they will allow us to improve the low-gain statistics for the $\alpha$-versus-gain curve (Fig. 9), and they may provide clues to the evolution of the PHD.

\section{Determination of $\alpha$ using PEACE-WHISPER density comparisons}

Since it is no longer possible to calibrate the MCP sensitivity in flight using the method described in Sect. 3, we have adopted an alternative procedure in which we adjust the $\alpha$ 

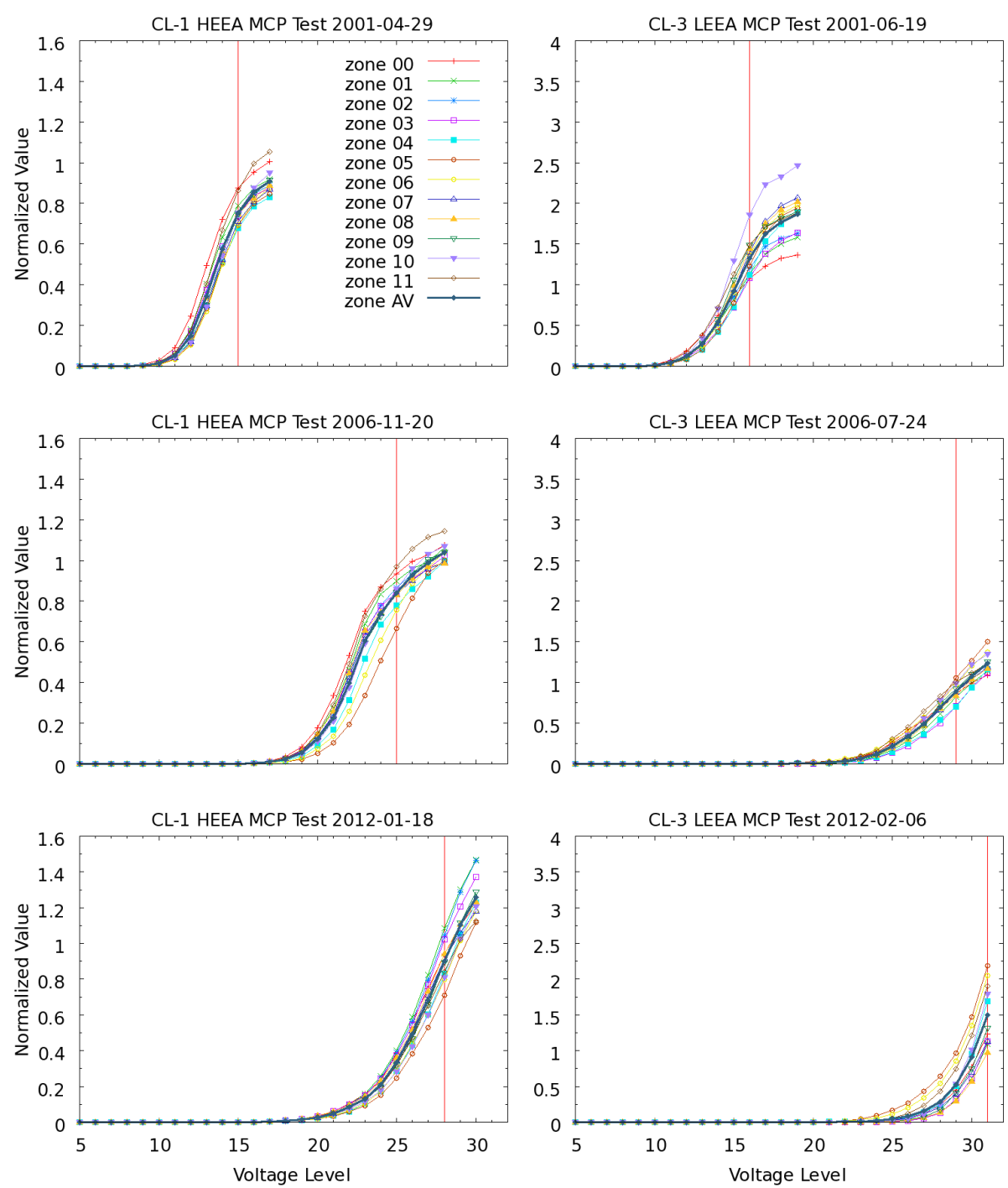

Fig. 11. Failure of the MCP test method. These plots show how the weekly MCP test results have evolved for Cluster-1 HEEA and Cluster3 LEEA over the mission. The red vertical line shows the operational voltage level of the test sensor used around the time of the test. The normalisation becomes unreliable if the operating voltage of the monitoring sensor is not sufficient to give $\alpha \sim 1$ for that sensor. This problem is clearly apparent in some cases, for example C3 LEEA in 2012.

factor in order to achieve agreement in electron density values from PEACE LEEA sensors with results from the WHISPER experiment (Décréau et al., 2001). This is only possible because of the high quality of the WHISPER total density data, optimised in active sounding mode, available in some of the plasma environments visited by Cluster.

The calibrations are extended to the HEEA sensors by comparing the densities from the HEEA and LEEA sensors in the energy overlap region. This method has so far been applied for the period November 2004 through to January 2012. This includes an overlap period with the results from in-orbit MCP tests, providing a check on the accuracy of our PEACEonly technique, which would be relevant in future missions with no sounder.

\subsection{LEEA sensors: LEEA-WHISPER density comparisons}

For the WHISPER densities we use WHISPER active mode electron data from the magnetosheath, available from the Cluster Active Archive (CAA). Corresponding PEACE LEEA electron densities are produced using the ground calibration geometric factor. To calculate electron densities from PEACE data also requires knowledge of the spacecraft potential. In this analysis the EFW (Electric Field and Wave experiment) (Gustafsson et al., 2001) spin resolution probespacecraft potential from the CAA is used. A correction of $+1 \mathrm{eV}$, which is suitable in the dense magnetosheath plasma environment, is applied to the EFW probe potential to give 
Cluster-2, 2001-12-06, 3DX1P LEEA + 3DX2P HEEA, (all anodes averaged)

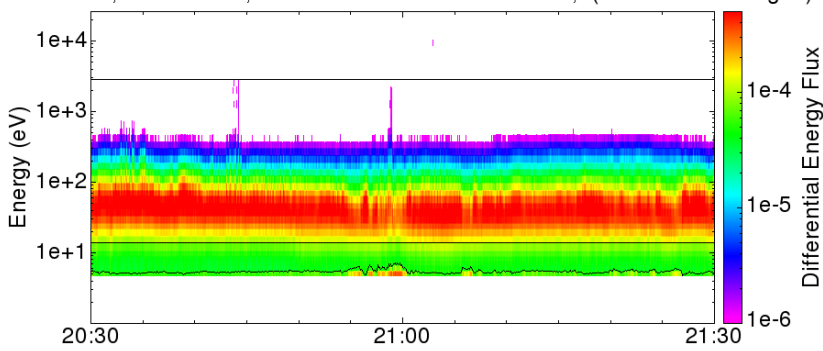

Cluster-2, 2003-04-08, 3DX1P LEEA + 3DX2P HEEA, (all anodes averaged)

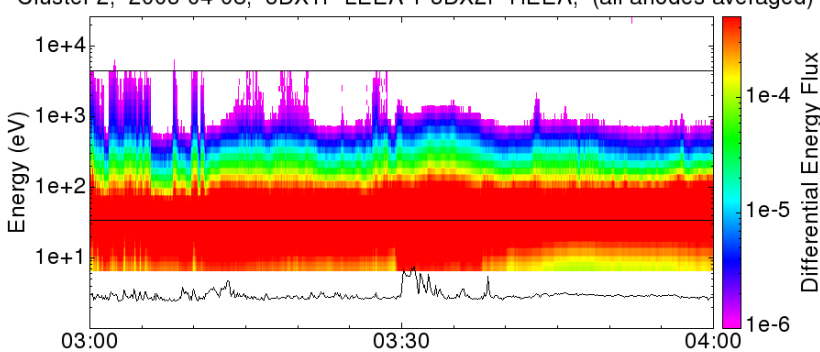

Fig. 12. Example of full coverage (top panel) and partial coverage (bottom panel) of the plasma energy range. The two black horizontal lines show the energy overlap region of the two PEACE sensors. Energies above the top black line are being measured by the HEEA sensor only. Energies below the bottom black line are being measured by the LEEA sensor only. Energies in between the two lines are measured by both sensors. If LEEA does not measure energies as low as the spacecraft potential we have "partial coverage".

the true value of the spacecraft electric potential. When calculating PEACE moments we try to eliminate photoelectrons in the plasma distribution by increasing the lower cut-off in the energy integration. We reject the energy bin containing the EFW probe-spacecraft potential and the one above as the $1 \mathrm{eV}$ correction mentioned above may put the true spacecraft potential in this bin. Sometimes "spikes" can be seen in the PEACE moments time series data. These "spikes" are observations of photoelectrons that are briefly energised by a few eV during WHISPER soundings, which occur periodically, at intervals of $52 \mathrm{~s}$ or $104 \mathrm{~s}$. PEACE moments data were not filtered for WHISPER soundings in this study because the contribution from "spikes" is not significant in the magnetosheath regions, compared to the very high plasma electron fluxes observed there at the same energies. In contrast, the additional flux associated with spikes in regions with lower plasma electron fluxes, such as the magnetotail plasmasheet, does significantly add to the plasma electron fluxes at low energy and hence to the phase space density, leading to clear variation in the moments. However, data from such regions was not used in this study.

\subsubsection{Event selection and partial coverage}

For this study carefully selected magnetosheath intervals are used. These are only available between November and June each year. Thus MCP degradation during the magnetotail crossing region (July to October) is not determined, however the decline is less severe in the magnetotail. It is important to determine the total electron density, thus we require that the energy range selected for the PEACE LEEA sensors is such that the sensor measures all of the plasma. If this is not the case then we would naturally expect the PEACE partial densities to be smaller than WHISPER densities. An example of this is shown in Fig. 12. In the upper spectrogram the LEEA sensor is measuring the energy range $4.7-2880.0 \mathrm{eV}$. It can be seen from the spectrogram that the LEEA sensor is measuring all energies above the spacecraft potential thus seeing all of the plasma, so this event would be selected for use in the study. In the lower spectrogram the LEEA sensor does not measure below $9.5 \mathrm{eV}$ however the spacecraft potential is $\sim 3 \mathrm{eV}$, so LEEA does not measure some of the plasma fluxes above the spacecraft potential which is expected to result in underestimated densities. Events of this kind are not used in our study. Useful events are selected manually by looking through spectrograms similar to those in Fig. 12.

\subsubsection{Filtering out possible errors in WHISPER \& EFW data}

Compromised points in both EFW and WHISPER data which could cause errors in the analysis are filtered out by using comparisons of these data. It is assumed that there should be a characteristic curve relating the EFW probe-spacecraft potential data and the WHISPER density data as shown by Pedersen et al. (2008). Figure 13 shows a plot of this type using selected events of Cluster-4 magnetosheath data between November 2002 and June 2003. Any points that are far from the trend are not used in the study.

\subsubsection{Determination of alpha}

A time history of the $\alpha$ factor with weekly time steps is obtained by extracting the peak (modal) value of weekly averaged LEEA / WHISPER density ratios. In order to produce daily values we interpolate between weekly points to give an $\alpha$ factor for each day. Special attention is paid to times when there is expected to be a sharp rise or fall in the $\alpha$ factor, such as when there is an MCP level raise or a thruster firing, by looking in detail at the density ratios of individual events instead of using the weekly average and interpolation technique. Also careful analysis is applied to the calibration intervals used for any dubious events which do not follow the $\alpha$ factor trend and which are deemed not to be real.

For the magnetotail seasons where WHISPER density data is not routinely available, the $\alpha$ factor is obtained by linear interpolation between the two values on either side of the gap, accounting for any MCP level raises and thrusters firings during these intervals where possible. Cross-calibrations of PEACE densities with WBD (Wide Band Data receiver) (Gurnett et al., 2001) densities in the plasmasheet have been 
Cluster-4, Nov 2002 - Jun 2003, Region-Sheath
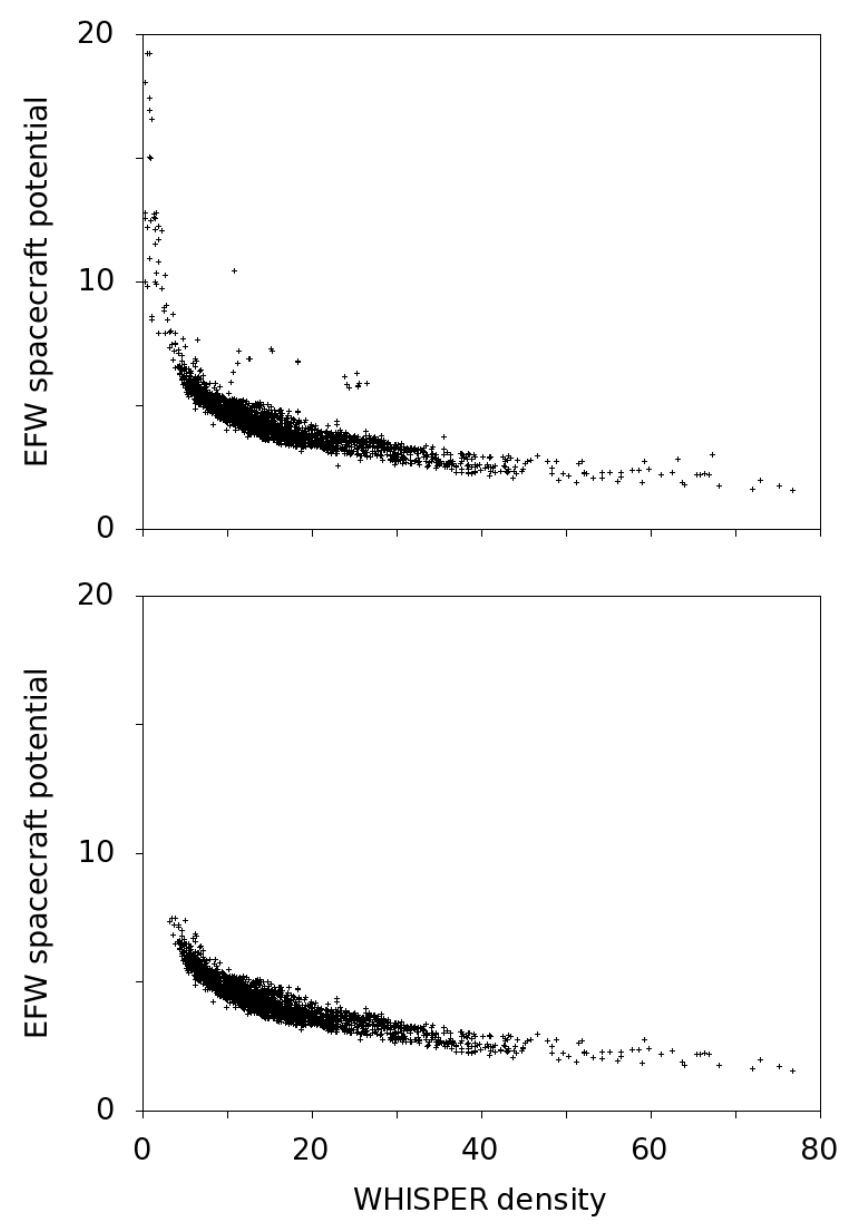

Fig. 13. Correlation between EFW spacecraft potential and WHISPER density for Cluster-4 using magnetosheath data between November 2002 and June 2003. Points which do not fit the trend (top plot) are removed and not included in the study (bottom plot).

used, where available, to validate these $\alpha$ factors. However there are very few intervals available for these studies. For the most part agreement was found. Disagreement for Cluster-4 in the 2007 tail season allowed us to fine-tune alpha factors which were inaccurate due to MCP gain degradation following thruster firings at the end of the tail season.

The $\alpha$ factors extracted from these density ratios apply for the MCP voltage level used at the time for the magnetosheath intervals (often the nominal $V_{\text {sheath }}=V_{\text {op }}-1$ level). The $\alpha$ factors for the normal operational and other voltage levels are then calculated by using the gain-versus-voltage and alphaversus-gain curves described in Sect. 3.

\subsection{HEEA sensors: HEEA-LEEA comparisons}

To calibrate the HEEA sensors PEACE HEEA densities are compared with PEACE LEEA densities calculated using data only from the energy overlap region of the two sensors. It

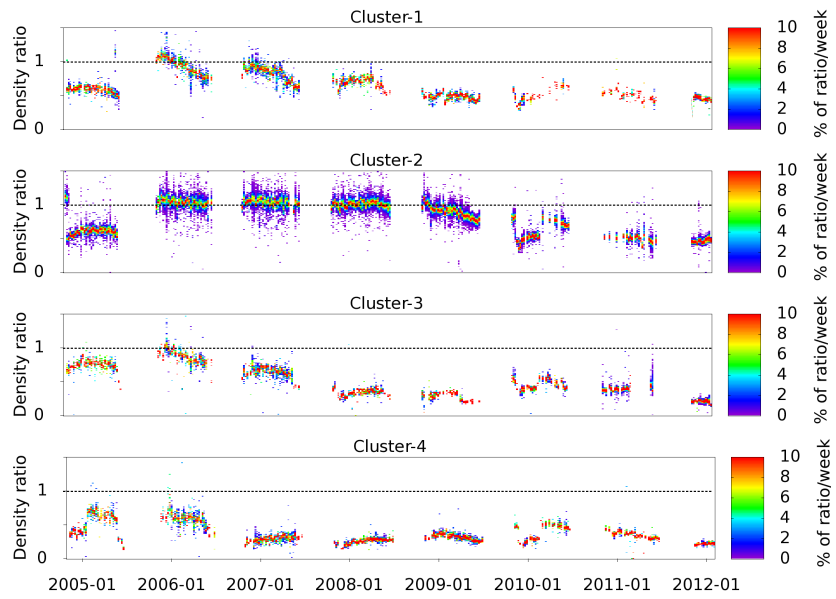

Fig. 14. Degradation of the four LEEA sensors between November 2004 and January 2012. These plots show the PEACE LEEA/WHISPER density ratio frequency. Each vertical strip of data is the frequency histogram for one week's worth of data.

is necessary to filter the events used in the study and reject those cases where the energy overlap of the sensors is small and/or the energy overlap covers tenuous plasmas which result in poor counting statistics. Poor count rates are becoming more common in later years as the MCP gains decrease. The PEACE HEEA densities are produced with only the ground calibration geometric factor applied. The PEACE LEEA densities are produced after applying the $\alpha$ correction factor determined from PEACE-WHISPER density comparisons.

\subsection{Results}

\subsubsection{LEEA sensitivity degradation}

The PEACE LEEA / WHISPER density ratios from November 2004 to January 2012 for each LEEA sensor are shown in Fig. 14. Each vertical strip in the plots is the frequency histogram of the density ratio for one week of data. These plots effectively show the sensitivity degradation of the LEEA sensors over this time period. The regular gaps in the plots are for the magnetotail months (July-October) where we do not see the magnetosheath. There is also a gap in the data for Cluster-3 between March and May 2011 where the Wave Experiment Consortium (WEC) instrument suite, which includes WHISPER and EFW, was non-operational.

Figure 15 shows the $\alpha$ factor history for the four LEEA sensors for November 2004 to January 2012. The $\alpha$ factors determined from both the in-orbit MCP test method (black) and PEACE-WHISPER density comparison method (red) are shown. By design, the alpha factors determined from PEACE-WHISPER comparisons include the correction required to refine the value of the time-independent $\varepsilon_{0}$ calibration parameter determined in ground tests (see Sect. 2). The correction to $\varepsilon_{0}$ is not included in the alpha obtained 

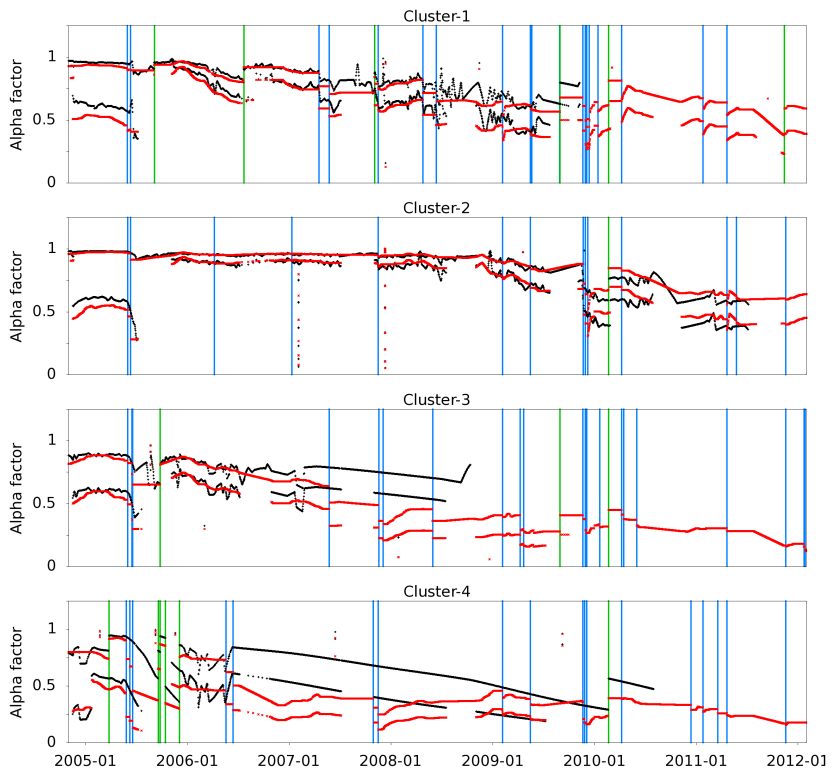

Fig. 15. The relative MCP sensitivity time history for the four LEEA sensors. The red points are the alpha factors inferred from the PEACE-WHISPER density comparison method including estimates in the magnetotail months. The black points are the alpha factors produced using the weekly MCP test method. For each data set the upper line is for operational MCP voltage level and the lower line for lowered MCP voltage level (used in high-flux environments). Commanded MCP voltage level changes are shown by green vertical lines. Thruster firings are shown by blue vertical lines.

from in-orbit MCP tests and is applied separately. Therefore in order to compare like with like alpha factors from the two methods, the correction for $\varepsilon_{0}$ has been factored out of the alpha factor from PEACE-WHISPER comparisons.

For each set of $\alpha$ factors the upper line is the $\alpha$ factor for the normal operational level and the lower line is the $\alpha$ factor for the lowered MCP voltage levels used in the magnetosheath and solar wind. MCP level raises (green vertical lines) and the thruster firings (blue vertical lines) which have had an observable impact on the $\alpha$ factor are also indicated. Very good agreement between the two methods is seen for the earlier years at normal operational voltage level. The weekly MCP test method is available in all months, unlike the LEEA-WHISPER comparison method which relies on observations in the magnetosheath. It can be clearly seen where the in-orbit MCP test method begins to fail for each sensor.

\subsubsection{HEEA sensitivity degradation}

The PEACE HEEA/PEACE LEEA density ratios from November 2004 to January 2012 for each HEEA sensor are shown in Fig. 16. The data shown in these plots are from intervals where both sensors are operating at their normal operational level (i.e. not lowered). These plots show the degrada-

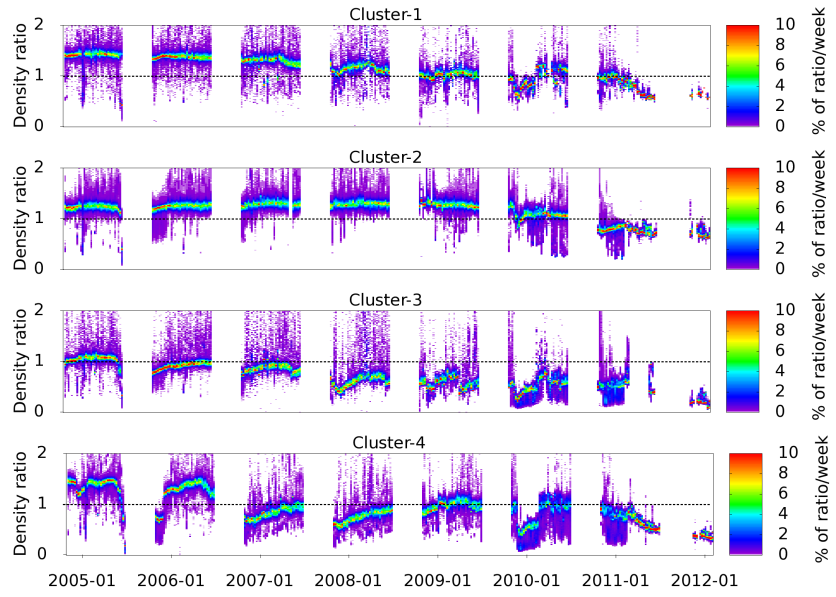

Fig. 16. Degradation of the four HEEA sensors between November 2004 and January 2012. These plots show the PEACE HEEA / PEACE LEEA density ratio frequency. Each vertical strip of data is the frequency histogram for one week's worth of data.

tion of the HEEA sensor efficiencies over this time period. As with the corresponding plot for the LEEA sensors, changes in the density ratio due to MCP voltage level raises and thrusters firings can be seen in the plots.

The $\alpha$ factor history for the four HEEA sensors extracted from the density ratio results are shown in Fig. 17 together with the $\alpha$ factors determined using the in-orbit method.

\subsubsection{Discussion}

There are several features that can be seen in these plots which require further discussion:

1. MCP operational voltage level raises: due to decline in MCP efficiency over time, the operational MCP voltage levels on all sensors have been raised at various times throughout the mission in order to recover a desired sensitivity level. These MCP voltage level changes have occurred at different times for different sensors, with some sensors requiring more level raises than others. We tend to aim for $\alpha \sim 0.5$ to 1 since the degradation rate has been observed to be greater when $\alpha>1$. In Figs. 14 and 15 an example of this level raise can be seen on 20 February 2010 where the voltage level was raised on all four LEEA sensors. The increase in gain/sensitivity is clearly evident.

2. Thruster firings: spacecraft thruster firings of a variety of durations have been performed throughout the mission. There is clear evidence that PEACE MCP sensitivity declines following some thruster firings. For example, firings which have had severe effects on the sensitivity can be seen in Figs. 15 and 17 in November 2007 and November/December 2009. However it has been observed that after some firings the MCP 
sensitivity does recover somewhat and in some cases to pre-firing levels. A separate study into the effect of thruster firings on the MCP efficiency is ongoing.

3. MCP voltage level lowering: from November 2003 the MCP voltage levels were routinely lowered by 1 or 2 levels when operating in the magnetosheath or solar wind as it was believed that the performance decline would be more gradual if the MCPs were operated at a lower gain when in high-flux environments. As of November 2009 the MCP voltage levels on Cluster3 and Cluster-4 are no longer lowered as their performance at the operational level from this time was considered as "low gain", so there was no need to lower them further. The increase in the density ratio in Fig. 14 for Cluster-3 and Cluster-4 at the start of the 2009/2010 dayside is due to this change in commanding of the voltage level and is not a real sensitivity increase.

4. MCP voltage lowering to estimated half gain: during the 2004/2005 dayside season the MCP voltage levels were lowered in order to achieve an estimated gain $=0.5 \times 10^{6}$ electrons on the sensors. This was performed to protect the MCPs as described in point 3 above. However the gains went lower than intended, so the attempted targeting of a preferred gain was not repeated.

\subsection{Limitations of the PEACE-WHISPER density comparison method}

There are several issues which cause problems for this method:

1. Availability of good magnetosheath calibration intervals for LEEA/WHISPER work: this is sometimes a major problem. Between November 2003 and June 2008 the PEACE sensors on only one spacecraft would operate throughout the magnetosheath and solar wind with the other spacecraft sensors powered off except during predicted times of bow shock and magnetopause crossings. The "observational spacecraft" role rotated between Cluster-1, 2 and 4 (only Cluster- 1 and 2 between March 2005 and June 2008). From November 2008 Cluster- 2 was always, and still is, used as the observational spacecraft with Cluster-1, 3 and 4 routinely turned off in the magnetosheath except around the bow shock and magnetopause crossings. For a short period between January and June 2012 Cluster1 was used as the observational spacecraft instead of Cluster-2. Thus for the non-observational spacecraft only magnetosheath data collected during bow shock and magnetopause crossings are available for the study.
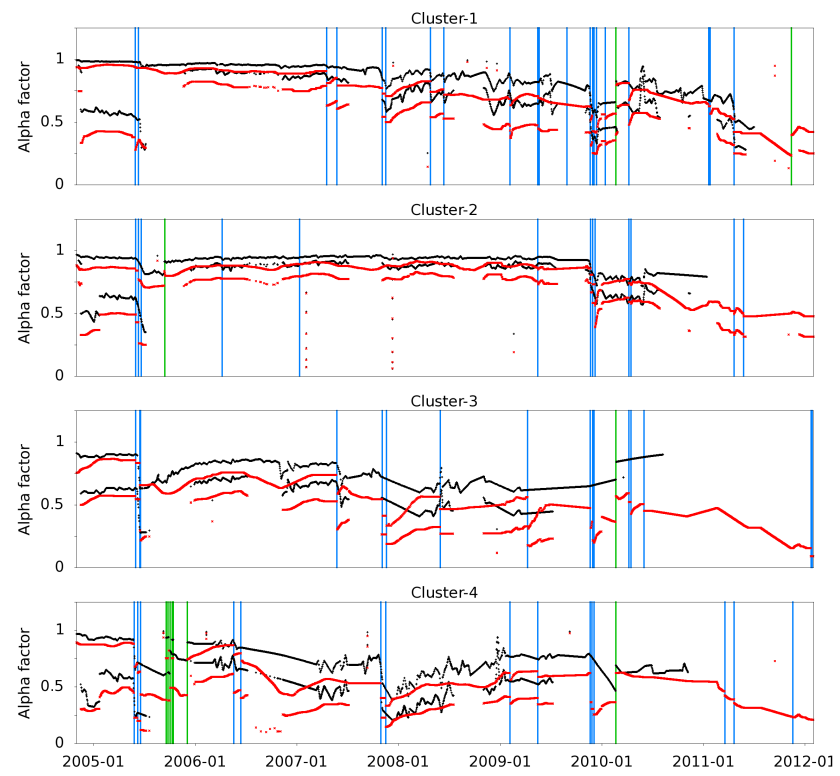

Fig. 17. The relative MCP sensitivity time history for the four HEEA sensors. The red points are the alpha factors inferred from HEEA-LEEA density comparisons. The black points are the alpha factors produced using the weekly MCP test method. For each data set the upper line is for operational MCP voltage level and the lower line for lowered MCP voltage level (used in high-flux environments). Commanded MCP voltage level changes are shown by green vertical lines. Thruster firings are shown by blue vertical lines.

2. Partial coverage of the plasma velocity distribution: even when the sensors are on in the magnetosheath not all intervals can be used. Depending on the energy range measured by the LEEA sensor, which is chosen to try and avoid the high fluxes of the photoelectrons, partial coverage of the plasma velocity distribution (see Sect. 4.1.1) can be a major problem too. This was particularly an issue in 2009/2010.

3. HEEA/LEEA energy overlap: a small energy overlap region can cause large spreads in the ratios. As a result many intervals need to be eliminated from the study.

4. Low counting statistics: this can also cause large spreads in the ratios.

5. Low gains: at very low gains the gain-versus-voltage and alpha-versus-gain curves (Sect. 3) are not as accurate as for higher gains, so determining alpha for different MCP voltage levels carries some error, which is compounded when comparing HEEA and LEEA.

To help with points 1, 2 and 3, special calibration intervals have been introduced into the routine commanding since 2011, to ensure full plasma coverage in the magnetosheath for the LEEA sensor and large energy overlap region for the two sensors. 


\section{Conclusions}

It has been shown in this paper that it has been essential to perform continuous in-flight calibrations to monitor the health and correct for the evolution of the MCP detector performance on each of the eight PEACE sensors. This builds on careful ground calibration work to define many parameters that cannot be determined in flight. We have described two independent methods to determine the detector sensitivity variations. The LEEA-WHISPER comparison method relies on accurate density determination by WHISPER and PEACE. The dual-sensor MCP test method uses only PEACE data and is based on some assumptions which have been discussed. The MCP test method works in all months, unlike the LEEA-WHISPER comparison method which cannot be used in the magnetotail season. Although the MCP test method can no longer be applied, we continue to have good knowledge of $\alpha$ factor evolution thanks to PEACE-WHISPER cross-calibrations. The good agreement of the two methods in earlier years validates the PEACE-only method, which may be of interest for future missions that do not carry a sounder.

Acknowledgements. The authors thank ESA and UKSA/STFC for funding the team, members of other PI teams for providing data required to carry out the work reported here and also the CAA team at ESTEC for supporting cross-calibration activities. We would also like to thank Dave Walton and Barry Hancock for their valuable technical assistance.

Edited by: A. Masson

\section{References}

Carlson, C. W., Curtis, D. W., Paschmann, G., and Michael, W.: An Instrument for Rapidly Measuring Plasma Distribution Functions with High Resolution, Adv. Space Res., 2, 67-70, doi:10.1016/0273-1177(82)90151-X, 1982.

Décréau, P. M. E., Fergeau, P., Krasnoselskikh, V., Le Guirriec, E., Lévêque, M., Martin, Ph., Randriamboarison, O., Rauch, J. L., Sené, F. X., Séran, H. C., Trotignon, J. G., Canu, P., Cornilleau, N., de Féraudy, H., Alleyne, H., Yearby, K., Mögensen, P. B., Gustafsson, G., André, M., Gurnett, D. C., Darrouzet, F., Lemaire, J., Harvey, C. C., Travnicek, P., and Whisper experimenters (Table 1): Early results from the Whisper instrument on Cluster: an overview, Ann. Geophys., 19, 1241-1258, doi:10.5194/angeo-19-1241-2001, 2001.

Eberhardt, E. H.: Gain model for microchannel plates, Appl. Optics, 18, 1418-1423, 1979.

Fazakerley, A. N., Lahiff, A. D., Wilson, R. J., Rozum, I., Anekallu, C., West, M., and Bacai, H.: PEACE Data in the Cluster Active Archive, The Cluster Active Archive: Studying the Earth's Space Plasma Environment, 129-144, doi:10.1007/978-90-4813499-1_8, 2010a.
Fazakerley, A. N., Lahiff, A. D., Rozum, I., Kataria, D., Bacai, H., Anekallu, C., West, M., and Asnes, A.: Cluster-PEACE In-flight Calibration Status, The Cluster Active Archive: Studying the Earth's Space Plasma Environment, 281-299, doi:10.1007/97890-481-3499-1_19, 2010b.

Gurnett, D. A., Huff, R. L., Pickett, J. S., Persoon, A. M., Mutel, R. L., Christopher, I. W., Kletzing, C. A., Inan, U. S., Martin, W. L., Bougeret, J.-L., Alleyne, H. St. C., and Yearby, K. H.: First results from the Cluster wideband plasma wave investigation, Ann. Geophys., 19, 1259-1272, doi:10.5194/angeo-191259-2001, 2001.

Gustafsson, G., André, M., Carozzi, T., Eriksson, A. I., Fälthammar, C.-G., Grard, R., Holmgren, G., Holtet, J. A., Ivchenko, N., Karlsson, T., Khotyaintsev, Y., Klimov, S., Laakso, H., Lindqvist, P.-A., Lybekk, B., Marklund, G., Mozer, F., Mursula, K., Pedersen, A., Popielawska, B., Savin, S., Stasiewicz, K., Tanskanen, P., Vaivads, A., and Wahlund, J.-E.: First results of electric field and density observations by Cluster EFW based on initial months of operation, Ann. Geophys., 19, 1219-1240, doi:10.5194/angeo19-1219-2001, 2001.

Johnstone, A. D., Alsop, C., Burge, S., Carter, P. J., Coates, A. J., Coker, A. J., Fazakerley, A. N., Grande, M., Gowan, R. A., Gurgiolo, C., Hancock, B. K., Narheim, B., Preece, A., Sheather, P. H., Winningham, J. D., and Woodliffe, R. D.: PEACE: A Plasma Electron and Current Experiment, Space Sci. Rev., 79, 351-398, doi:10.1023/A:1004938001388, 1997.

Pedersen, A., Lybekk, B., André, M., Eriksson, A., Masson, A., Mozer, F. S., Lindqvist, P.-A., Décréau, P. M. E., Dandouras, I., Sauvaud, J.-A., Fazakerley, A., Taylor, M., Paschmann, G., Svenes, K. R., Torkar, K., and Whipple, E.: Electron density estimations derived from spacecraft potential measurements on Cluster in tenuous plasma regions, J. Geophys. Res., 113, A07S33, doi:10.1029/2007JA012636, 2008.

Rème, H., Aoustin, C., Bosqued, J. M., Dandouras, I., Lavraud, B., Sauvaud, J. A., Barthe, A., Bouyssou, J., Camus, Th., Coeur-Joly, O., Cros, A., Cuvilo, J., Ducay, F., Garbarowitz, Y., Medale, J. L., Penou, E., Perrier, H., Romefort, D., Rouzaud, J., Vallat, C., Alcaydé, D., Jacquey, C., Mazelle, C., d’Uston, C., Möbius, E., Kistler, L. M., Crocker, K., Granoff, M., Mouikis, C., Popecki, M., Vosbury, M., Klecker, B., Hovestadt, D., Kucharek, H., Kuenneth, E., Paschmann, G., Scholer, M., Sckopke, N., Seidenschwang, E., Carlson, C. W., Curtis, D. W., Ingraham, C., Lin, R. P., McFadden, J. P., Parks, G. K., Phan, T., Formisano, V., Amata, E., Bavassano-Cattaneo, M. B., Baldetti, P., Bruno, R., Chionchio, G., Di Lellis, A., Marcucci, M. F., Pallocchia, G., Korth, A., Daly, P. W., Graeve, B., Rosenbauer, H., Vasyliunas, V., McCarthy, M., Wilber, M., Eliasson, L., Lundin, R., Olsen, S., Shelley, E. G., Fuselier, S., Ghielmetti, A. G., Lennartsson, W., Escoubet, C. P., Balsiger, H., Friedel, R., Cao, J.-B., Kovrazhkin, R. A., Papamastorakis, I., Pellat, R., Scudder, J., and Sonnerup, B.: First multispacecraft ion measurements in and near the Earth's magnetosphere with the identical Cluster ion spectrometry (CIS) experiment, Ann. Geophys., 19, 1303-1354, doi:10.5194/angeo19-1303-2001, 2001. 